\title{
EFCC1 as a putative prognostic biomarker in lung adenocarcinoma
}

\author{
Shengnan Yu' ${ }^{1,2}$, Gao Zhang ${ }^{2}$ \\ ${ }^{1}$ Department of Oncology, Tongji Hospital of Tongji Medical College, Huazhong University of Science and Technology, Wuhan, China; \\ ${ }^{2}$ Department of Neurosurgery and The Preston Robert Tisch Brain Tumor Center, Duke University, Durham, NC, USA \\ Correspondence to: Gao Zhang. Department of Neurosurgery and The Preston Robert Tisch Brain Tumor Center, Duke University, Durham, NC, \\ USA. Email: gao.zhang@duke.edu. \\ Provenance and Peer Review: This article was commissioned by the editorial office, Annals of Translational Medicine. The article did not undergo \\ external peer review. \\ Comment on: Xia L, Zhu Y, Zhang C, et al. Decreased expression of EFCC1 and its prognostic value in lung adenocarcinoma. Ann Transl Med 2019;7:672.
}

Submitted Apr 20, 2020. Accepted for publication May 19, 2020.

doi: $10.21037 /$ atm-2020-93

View this article at: http://dx.doi.org/10.21037/atm-2020-93

Lung cancer is known for its high incidence worldwide. Meanwhile, it is the leading cause of cancer-related death (1). In 2018, estimated numbers of death for lung cancer is 690,567 in China and 152,423 in United States (2). Lung cancer is mainly divided into two subtypes: non-small cell lung cancer (NSCLC) and small cell lung cancer (SCLC). And NSCLC includes lung adenocarcinoma (ADC), squamous cell carcinoma (SQC) and large cell lung cancer, etc. Over the past few decades, multiple therapies have been developed to treat patients with NSCLC. The traditional therapies such as surgery, chemotherapy and radiotherapy have extended patients' survival to some extent. However, the benefits have always been limited and transient (3). For patients with recurrent or unresectable lung cancer, there remains an urgent need to develop more novel treatments. The small molecule tyrosine kinase inhibitors (TKIs) and immunotherapy have brought new hope to patients with NSCLC (4). But the TKIs are only beneficial for a subset of patients whose tumors harbor specific gene alterations, such as EGFR mutations, EML4-ALK rearrangements. And due to the acquisition of secondary mutations or activation of bypath signaling pathways, acquired resistance of TKIs become another inevitable challenge (5). The immune checkpoint blockade therapy is just suitable for some selected patients. When immune checkpoint inhibitor acts as monotherapy for lung cancer patients, its efficacy is low. In general, lung cancer patients with higher expression of immune checkpoint molecules (PD-L1, PD-1 or CTLA-4) in tumor tissues exhibited more obvious response to checkpoints inhibitors (6). Thus, early diagnosis and novel treatment options are critical for improving the outcome of patients with lung cancer. Specifically, it is essential to identify biomarkers that can be used to predict prognosis and to track recurrence.

EF-hand and coiled-coil domain containing 1 (EFCC1) is a protein coding gene that is located on chromosome 3q21.3. EFCC1, also called coiled-coil domain containing 48 (CCDC48), belongs to the CCDC family. Coiledcoil domain containing proteins exhibit a broad range of different functions associated with their specific coiledcoil motifs (7). Aberrant expression of coiled-coil domain containing proteins are related to the malignant behavior of human cancers. Studies reported that elevated expression of CCDC50S (a truncated oncogenic splice variant) in hepatocellular carcinoma (HCC) was positively correlated with tumor grade, clinical stage and poor prognosis. CCDC50S promotes HCC progression by activating the Ras signaling pathway (8). CCDC178 is upregulated in HCC and promotes the metastasis of HCC in vivo (9). Besides, some CCDC family members were also decreased in tumor tissues. Such as in pancreatic ductal ADC, CCDC68 was identified as a putative tumor suppressor gene (10). CCDC6, a tumor suppressor gene involved in apoptosis and DNA damage response, restored the resistance to cisplatinum and sensitized NSCLC cells to olaparib. CCDC6 is negatively correlated with the 
disease-free survival (DFS) and the overall survival (OS) in lung cancer (11). CCDC6 was associated with the clinicopathological status in papillary thyroid carcinoma and it reversed the effects of microRNA-146b-5p on cell proliferation, migration, invasion, and cell cycle both in vitro and in vivo (12). In addition, the binding of CCDC98 with breast cancer-1 (BRCA1) was involved in the mammalian DNA damage response (13). The interaction of Jumonji domain-containing protein 2A (JMJD2A) and CCDC8 sensitized gastric cancer to chemotherapy (14). However, the function of EFCC1 in cancer remains unknown which warrants further studies to explore the role of EFCC1 in cancer.

The authors first carried out the bioinformatics analysis to analyze RNA sequencing (RNAseq) data of normal lung, early-stage lung ADC, and advanced-stage lung ADC samples from The Cancer Genome Atlas (TCGA) database, which includes a variety of genomics data from 33 cancer types. The authors found that EFCC1 was one of top ten genes that are significantly down-regulated in lung ADC patients (15). From the Human Protein Atlas website (https://www.proteinatlas.org/), we found that the expression of EFCC1 at the mRNA level is markedly higher in normal lung tissue than other tissues (Figure 1A). The immunohistochemical staining of normal lung tissue showed that high expression of EFCC1 was located in cytoplasm and membrane (Figure 1B). And the EFCC1 was expressed in lung ADC but not in SQC (Figure 1C). Compared with other cancer types such as prostate cancer, liver cancer, colorectal cancer and breast cancer, the expression of EFCC1 at the protein level is highest in lung ADC (Figure 1C). Although we do not yet know the exact function of EFCC1 in lung cancer, the predominant expression of EFCC1 in normal lung tissue suggested that it could serve as a putative biomarker. The expression of EFCC1 at the mRNA level was sharply decreased in lung ADC samples, which may be associated with the DNA methylation level of EFCC1 (15). Besides, the expression level of EFCC1 is inversely correlated with the clinical tumor stage. The authors performed the survival analysis and showed that lung ADC patients with lower expression of EFCC1 at the mRNA level had a shorter OS (15). We also performed the Kaplan-Meier survival analysis of patients with lung cancer, lung ADC and lung SQC based on the expression of EFCC1 (http://kmplot.com/ analysis/index.php? $\mathrm{p}=$ service\&cancer=lung) (Figure 2). It is well known that NSCLC accounts for the majority of lung cancer, and lung $\mathrm{ADC}$ and SQC are more common in
NSCLC. Therefore, we mainly evaluated the relationship between EFCC1 expression level and OS or time to first progression (FP) in lung $\mathrm{ADC}$ and SQC. We found that lower expression EFCC1 predicted unfavorable OS and FP in lung cancer. And the trend is more pronounced in lung ADC but not in SQC. The lower expression of EFCC1 suggested shorter OS and quicker progression in lung ADC.

The authors validated EFCC1 expression in lung ADC and matched adjacent non-tumor tissues from five lung ADC patients by qRT-PCR and western blot assay. The expression of EFCC1, both at mRNA and protein levels, was significantly down-regulated in lung ADC compared with adjacent normal lung. Commercial tumor microarray (TMA) with 130 clinical lung ADC samples was probed with the EFCC1 antibody. Their data demonstrated that lower expression of EFCC1 was associated with tumor TNM stage and lymph node metastasis. The analysis of univariate and multivariate cox proportional hazards showed that lower expression EFCC1 was an unfavorable prognosis factor both in TCGA and TMA tumor tissues (15). Moreover, it is essential to collect peripheral blood samples from lung ADC patients and measure the level of EFCC1 at the serum. The peripheral blood samples should also include those from normal, lung ADC patients with different TNM stage and different grade. If the serum EFCC1 is correlated with the clinicopathological parameters, EFCC1 will become a potential non-invasive prognostic biomarker. At present, progastrin-releasing peptide (ProGRP) and neuron specific enolase (NSE) are classic tumor markers for diagnosis and prognosis of SCLC (16). And the carcinoembryonic antigen (CEA), cytokeratin 19 fragment (CYFRA 21-1) and squamous carcinoma antigen (SCCA) are usually used in the diagnosis and monitor of NSCLC (17). The sensitivity and specificity of these serum tumor markers are not as high as we expected. Therefore, these biomarkers can only be used as a reference index for the diagnosis of lung cancer and to evaluate the therapeutic effect. It is crucial to identify biomarkers with higher sensitivity and specificity.

In summary, the expression of EFCC1 was measured in tumor tissues from clinical lung ADC patients. And it was correlated with the metastasis to lymph node and tumor TNM stage. Lower expression level of EFCC1 predicted poor OS and FP. Therefore, EFCC1 could be a candidate biomarker for this malignancy. It is likely that EFCC1 is a bona fide tumor suppressor gene. And that warrants further investigation of the role of EFCC1 in regulation of tumorigenesis, invasion and migration, the function of EFCC1 under physiological conditions, and elucidation of 


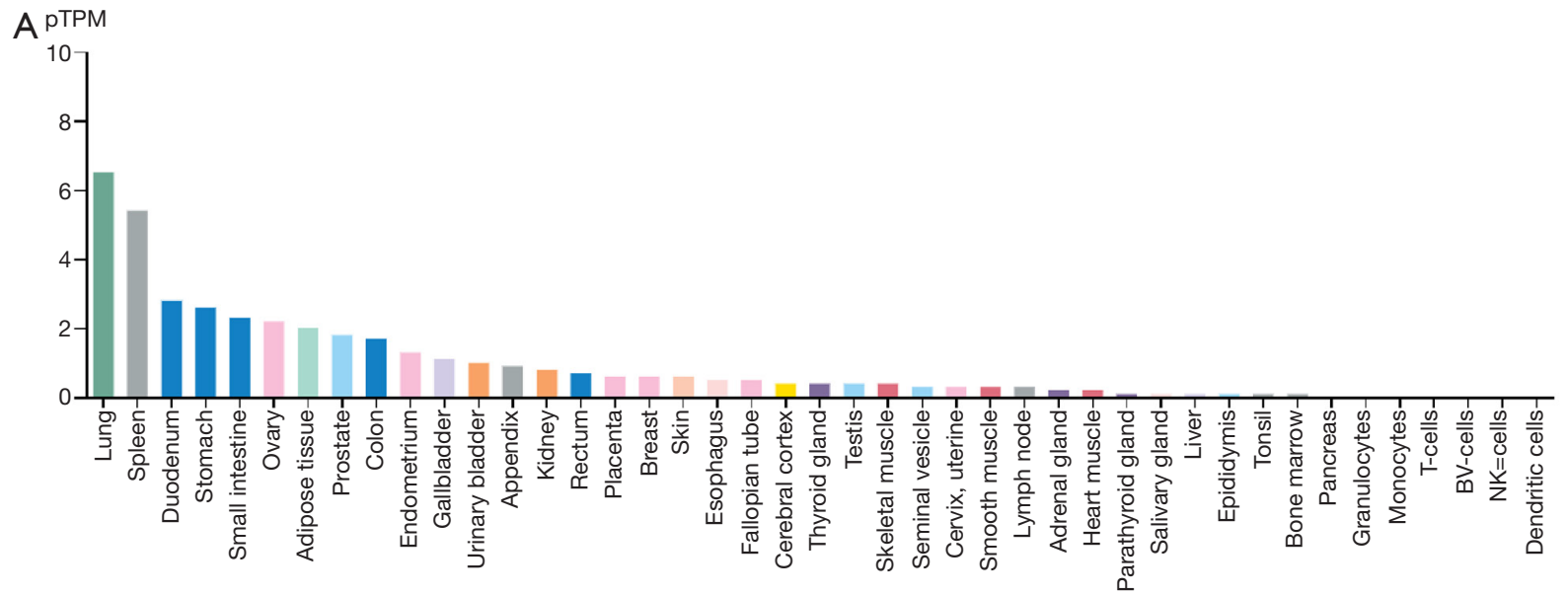

B

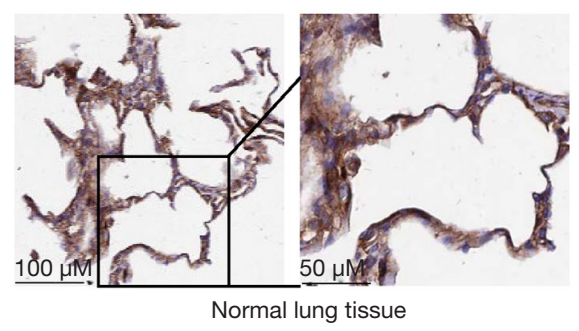

C

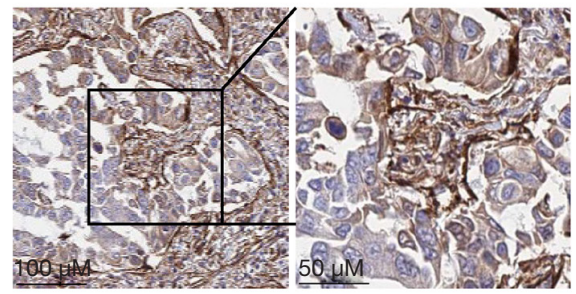

Lung cancer $(A D C)$

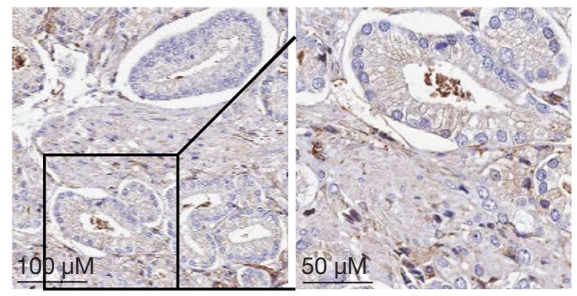

Prostate cancer

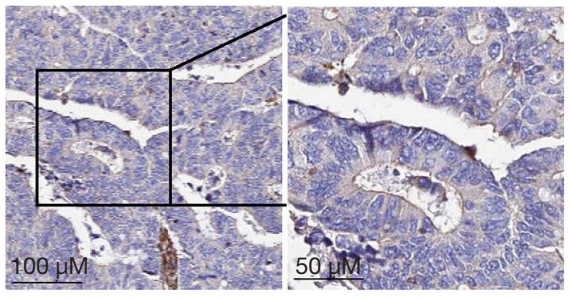

Colorectal cancer

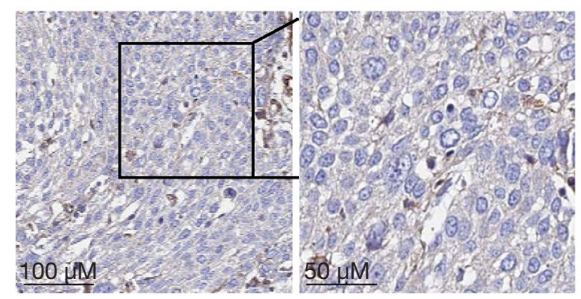

Lung cancer (SQC)

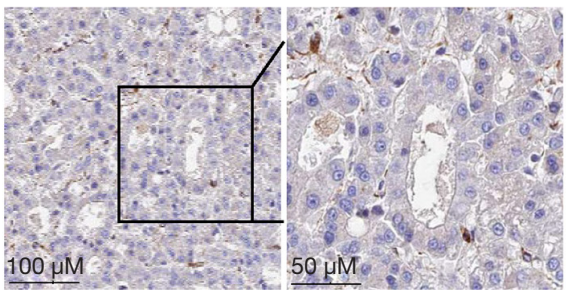

Liver cancer

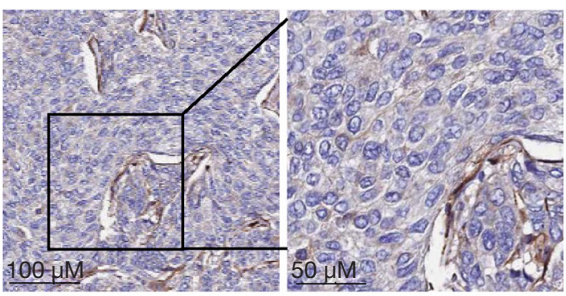

Breast cancer

Figure 1 The expression of EFCC1 in human normal and cancer tissues from the Human Protein Atlas website. (A) The mRNA level of EFCC1 in normal tissues, including blood cells. (B) The EFCC1 protein in normal lung tissue. (C) The expression of EFCC1 in different tumor tissues, including lung $\mathrm{ADC}$, lung SQC, prostate cancer, liver cancer, colorectal cancer and breast cancer. pTPM, protein-transcripts per million. ADC, adenocarcinoma; SQC, squamous cell carcinoma. 

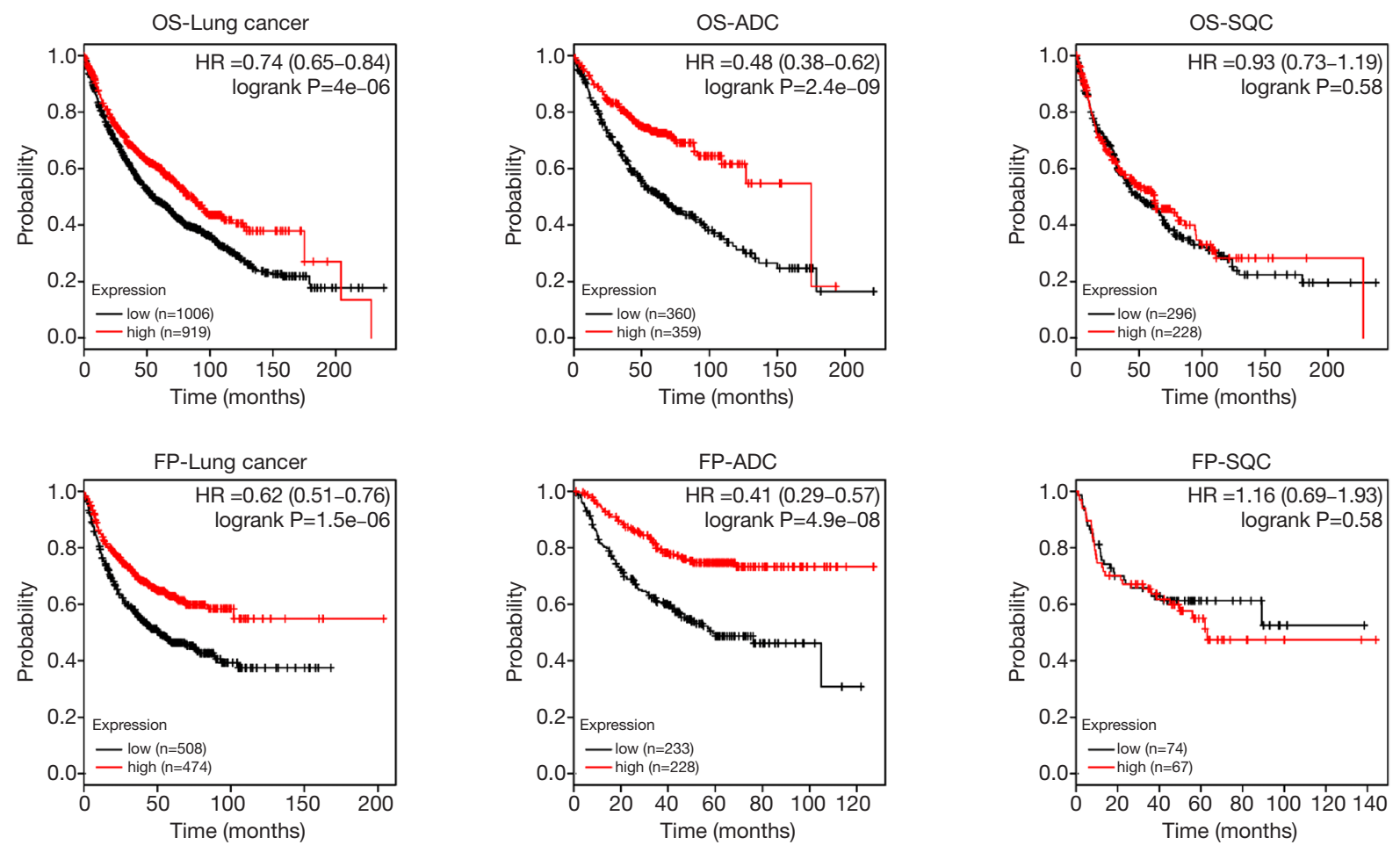

Figure 2 Low expression of EFCC1 is correlated with unfavorable OS and FP in lung ADC. Kaplan-Meier survival curves are from KaplanMeier Plotter website. OS, overall survival; FP, first progression; ADC, adenocarcinoma; SQC, squamous cell carcinoma.

signaling pathways that EFCC1 interact with.

\section{Acknowledgments}

Funding: None.

\section{Footnote}

Conflicts of Interest: Both authors have completed the ICMJE uniform disclosure form (available at http://dx.doi. org/10.21037/atm-2020-93). The authors have no conflicts of interest to declare.

Ethical Statement: The authors are accountable for all aspects of the work in ensuring that questions related to the accuracy or integrity of any part of the work are appropriately investigated and resolved.

Open Access Statement: This is an Open Access article distributed in accordance with the Creative Commons Attribution-NonCommercial-NoDerivs 4.0 International License (CC BY-NC-ND 4.0), which permits the non- commercial replication and distribution of the article with the strict proviso that no changes or edits are made and the original work is properly cited (including links to both the formal publication through the relevant DOI and the license). See: https://creativecommons.org/licenses/by-nc-nd/4.0/.

\section{References}

1. Siegel RL, Miller KD, Jemal A. Cancer statistics, 2020. CA Cancer J Clin 2020;70:7-30.

2. Feng RM, Zong YN, Cao SM, et al. Current cancer situation in China: good or bad news from the 2018 Global Cancer Statistics? Cancer Commun (Lond) 2019;39:22.

3. Postmus PE, Kerr KM, Oudkerk M, et al. Early and locally advanced non-small-cell lung cancer (NSCLC): ESMO Clinical Practice Guidelines for diagnosis, treatment and follow-up. Ann Oncol 2017;28:iv1-21.

4. Herbst RS, Morgensztern D, Boshoff C. The biology and management of non-small cell lung cancer. Nature 2018;553:446-54.

5. Jia Y, Yun CH, Park E, et al. Overcoming EGFR(T790M) and EGFR(C797S) resistance with mutant-selective 
allosteric inhibitors. Nature 2016;534:129-32.

6. Osmani L, Askin F, Gabrielson E, et al. Current WHO guidelines and the critical role of immunohistochemical markers in the subclassification of non-small cell lung carcinoma (NSCLC): Moving from targeted therapy to immunotherapy. Semin Cancer Biol 2018;52:103-9.

7. Burkhard P, Stetefeld J, Strelkov SV. Coiled coils: a highly versatile protein folding motif. Trends Cell Biol 2001;11:82-8.

8. Wang H, Zhang CZ, Lu SX, et al. A Coiled-Coil Domain Containing 50 Splice Variant Is Modulated by Serine/Arginine-Rich Splicing Factor 3 and Promotes Hepatocellular Carcinoma in Mice by the Ras Signaling Pathway. Hepatology 2019;69:179-95.

9. Hu X, Zhao Y, Wei L, et al. CCDC178 promotes hepatocellular carcinoma metastasis through modulation of anoikis. Oncogene 2017;36:4047-59.

10. Radulovich N, Leung L, Ibrahimov E, et al. Coiled-coil domain containing 68 (CCDC68) demonstrates a tumorsuppressive role in pancreatic ductal adenocarcinoma. Oncogene 2015;34:4238-47.

11. Morra F, Luise C, Visconti R, et al. New therapeutic perspectives in CCDC6 deficient lung cancer cells. Int J

Cite this article as: Yu S, Zhang G. EFCC1 as a putative prognostic biomarker in lung adenocarcinoma. Ann Transl Med 2020;8(16):981. doi: 10.21037/atm-2020-93
Cancer 2015;136:2146-57.

12. Jia M, Shi Y, Li Z, et al. MicroRNA-146b-5p as an oncomiR promotes papillary thyroid carcinoma development by targeting CCDC6. Cancer Lett 2019;443:145-56.

13. Kim H, Huang J, Chen J. CCDC98 is a BRCA1-BRCT domain-binding protein involved in the DNA damage response. Nat Struct Mol Biol 2007;14:710-5.

14. Nakagawa T, Sato Y, Tanahashi T, et al. JMJD2A sensitizes gastric cancer to chemotherapy by cooperating with CCDC8. Gastric Cancer 2020;23:426-36.

15. Xia L, Zhu Y, Zhang C, et al. Decreased expression of EFCC1 and its prognostic value in lung adenocarcinoma. Ann Transl Med 2019;7:672.

16. Shibayama T, Ueoka H, Nishii K, et al. Complementary roles of pro-gastrin-releasing peptide (ProGRP) and neuron specific enolase (NSE) in diagnosis and prognosis of small-cell lung cancer (SCLC). Lung Cancer 2001;32:61-9.

17. Wu H, Wang Q, Liu Q, et al. The Serum Tumor Markers in Combination for Clinical Diagnosis of Lung Cancer. Clin Lab 2020. doi: 10.7754/Clin.Lab.2019.190533. 